\title{
A Bit-Labeling Design for Trellis-Shaped Single-Carrier PSK with PAPR Reduction
}

\author{
Yuuki Nishino, Makoto Tanahashi, and Hideki Ochiai \\ Department of Electrical and Computer Engineering, Yokohama National University, 79-5 Tokiwadai, Hodogaya, Yokohama, \\ Kanagawa 240-8501, Japan \\ Correspondence should be addressed to Hideki Ochiai, hideki@ynu.ac.jp
}

Received 15 May 2010; Revised 23 July 2010; Accepted 31 August 2010

Academic Editor: Naofal Al-Dhahir

Copyright ( 92011 Yuuki Nishino et al. This is an open access article distributed under the Creative Commons Attribution License, which permits unrestricted use, distribution, and reproduction in any medium, provided the original work is properly cited.

Band-limited single-carrier signals, even with a phase-shift keying (PSK) constellation, suffer from relatively high peak-to-average power ratio (PAPR) when a narrow pulse-shaping filter is used at the transmitter. In our recent work, an application of trellis shaping (TS) has been studied extensively for the purpose of reducing PAPR of band-limited single-carrier PSK signals, and it has been shown that a nearly constant envelope signal can be generated even with the use of nearly rectangular pulse-shaping filter. In this paper, we first demonstrate that the uncoded bit error rate (BER) and PAPR reduction capability of the TS considerably depend on the bit labeling. We then propose a new bit labeling for high-order PSK constellation that can efficiently reduce PAPR while achieving BER performance comparable to that of Gray labeling. Finally, the BER of each constellation is theoretically analyzed and compared with the simulation results.

\section{Introduction}

Power and bandwidth efficiencies have been two important factors in wireless communications systems. In general, strictly band-limited signals suffer from high peak-toaverage power ratio (PAPR). Linear amplification of high PAPR signals requires a large backoff operation of power amplifier, which results in a considerable penalty in terms of power efficiency. Therefore, there is a tradeoff between bandwidth efficiency and power consumption at the transmitter.

Single-carrier signals have been adopted for the uplink of next generation communications [1] mainly due to its low PAPR property compared to multicarrier signals. However, if the bandwidth of the generated signal is severely limited by the use of narrow pulse-shaping filter, its envelope also experiences considerable fluctuation, resulting in high PAPR. Therefore, in this case, reduction of PAPR is also critical.

A PAPR problem of single-carrier systems has been studied and several PAPR reduction schemes have been proposed in the literature [2-5]. For example, in [2], an adaptive peak suppression scheme is proposed for controlling peak power of band-limited PSK signals. This is achieved by introducing intentional amplitude fluctuation, which causes some loss in terms of required average power [2]. More recently, in [4], PAPR properties of single-carrier frequency division multiple access (SC-FDMA) signal have been studied in the framework of space-frequency block codes (SFBC) and a mapping scheme that can avoid additional PAPR increase due to the use of SFBC has been proposed. In [5], PAPR properties of continuous phase modulation (CPM) combined with single-carrier frequency domain equalization (SC-FDE) have been investigated and an approach for maintaining phase continuity that guarantees constant envelope is proposed.

The trellis shaping (TS) [6] is a constellation-shaping method that can control symbol sequences by using the structure of convolutional (or trellis) codes and the Viterbi algorithm (VA) at the transmitter. Originally, TS was introduced in order to reduce the average power of quadrature amplitude modulation (QAM) signals by controlling symbol sequences. Later, its application to PAPR reduction of a single-carrier system was studied in [7]. More recently, in [8], it has been shown that a carefully designed TS is capable of generating near constant envelope for PSK signaling even if a very narrow pulse-shaping filter is employed. 
In this paper, we focus on the TS approach proposed in [8], since it can achieve both high bandwidth efficiency and near constant envelope for single-carrier systems. Although its envelope is not strictly constant as that of CPM [5], it is a linear modulation and thus the complexity of the receiver will be lower than that of CPM with a comparable bandwidth efficiency.

The main objective of this paper is to reveal the effects of bit labeling on the performance of the TS with highorder PSK constellations. As is well known, Gray labeling is preferable for uncoded PSK, since it minimizes the number of the bit errors associated with the dominant symbol error event and thus can minimize the bit error rate (BER). However, as we will demonstrate, Gray labeling may not be suitable for effective PAPR reduction based on the TS. On the other hand, natural labeling turns out to be effective for PAPR reduction, but it results in poorer BER performance compared to that of Gray mapping. Therefore, we propose a new bit labeling, referred to as a double-Gray (D-Gray) labeling, that can balance the two labelings. This new labeling is able to efficiently reduce PAPR while achieving BER performance comparable to that of Gray labeling. We confirm the effectiveness of the new labeling based on the theoretical BER analysis together with computer simulations.

We note that bit labeling itself has been extensively studied in the literature; to list a few recent examples, optimization of the bit labeling of various constellations has been studied in $[9,10]$. Joint optimization of signal constellation and bit labeling is proposed in [11] for bit-interleaved coded modulation with iterative decoding (BICM-ID) [12]. A constellation labeling that improves the error floor associated with iterative decoding is proposed in [13].

This paper is organized as follows. In Section 2, the principle of TS with PAPR reduction for single-carrier systems and its associated notations are briefly reviewed. Effects of bit labeling on the TS are studied and a new bit labeling is proposed in Section 3. Section 4 presents several simulation results that will confirm the effectiveness of the proposed labeling. The uncoded TS system generally causes performance degradation in terms of BER compared with that without shaping due to the syndrome operation at the receiver, and this effect is theoretically analyzed in Section 5. Finally, concluding remarks and future work are given in Section 6.

\section{Trellis Shaping}

Figure 1 is a general system model of trellis shaping, where $\mathbf{G}_{s}$ is a generator matrix of a convolutional code, $\mathbf{H}_{s}^{T}$ is the corresponding syndrome former matrix, and $\left(\mathbf{H}_{s}^{-1}\right)^{T}$ is its left inverse matrix. We assume that $\mathbf{G}_{s}$ is a $1 \times n_{s}$ matrix and thus $\mathbf{H}_{s}^{T}$ is an $n_{s} \times\left(n_{s}-1\right)$ matrix. The inverse matrix is thus an $\left(n_{s}-1\right) \times n_{s}$ matrix. These matrices should satisfy

$$
\mathbf{G}_{s} \mathbf{H}_{s}^{T}=\mathbf{0}, \quad\left(\mathbf{H}_{s}^{-1}\right)^{T} \mathbf{H}_{s}^{T}=\mathbf{I},
$$

where $\mathbf{0}$ denotes a zero vector and $\mathbf{I}$ is an identity matrix.
At the transmitter, an input information sequence $\mathbf{b}$ is divided into a sequence of shaping bits $\mathbf{s}$ and that of nonshaping bits $\mathbf{u}$. The shaping bits $\mathbf{s}$ are encoded by the left inverse of the syndrome former as

$$
\widetilde{\mathbf{s}}=\mathbf{s}\left(\mathbf{H}_{s}^{-1}\right)^{T} .
$$

This shaping process introduces one bit redundancy in $\mathbf{s}$ and at this stage, one may add arbitrary codeword of $\mathbf{G}_{s}$ to $\widetilde{s}$. Specifically, $\mathbf{v}=\mathbf{x G}_{\mathbf{s}}$ can be added in modulo-2 manner to $\widetilde{\mathbf{s}}$, where $\mathbf{x}$ is an arbitrary bit sequence. This sequence $\mathbf{z}=\widetilde{\mathbf{s}}+\mathbf{v}$ together with a sequence of nonshaping bits $\mathbf{u}$ may determine, based on some bit labeling, the resulting PSK symbol sequence $S=\left\{S_{l},-\infty<l<\infty\right\}$ to be transmitted. At the receiver, the received estimate symbol sequence $\widehat{S}$ is divided into $\hat{\mathbf{z}}$ and $\hat{\mathbf{u}}$, and the original shaping bits $\mathbf{s}$ can be recovered simply by multiplying $\hat{\mathbf{z}}$ with $\mathbf{H}_{s}^{T}$, since $\hat{\mathbf{z}} \mathbf{H}_{s}^{T}=\mathbf{s}$ as long as $\hat{\mathbf{z}}=\mathbf{z}[6]$.

The baseband signal at the transmitter is expressed as

$$
s(t)=\sum_{l=-\infty}^{\infty} S_{l} g\left(t-l T_{s}\right)
$$

where $T_{s}$ is the Nyquist symbol interval and $g(t)$ is the pulseshaping filter. Our objective is to choose $\mathbf{v}$ (or $\mathbf{x}$ ) such that the resulting dynamic range of the signal envelope, that is, $|s(t)|$, has a low peak value.

2.1. External Memories. For peak power reduction of linearly modulated and pulse-shaped signals, we have to consider not only the present symbol, but also the previously generated symbols, since the symbol dynamic range is determined by the multiple consecutive symbol patterns over the effective length of the impulse response of pulse shaping filter.

To this end, we introduce external memories [7] such that the past symbols can be taken into account for metric calculation of the peak power associated with the present symbol. As a specific example, let us consider the basic code generator in Figure 2(a). The corresponding code generator with $m_{\mathrm{ex}}=3$ external memories is depicted in Figure 2(b). By adding $m_{\mathrm{ex}}$ external memories, we can jointly consider the present symbol and the past $m_{\mathrm{ex}}$ symbols for codeword selection. By filtering the set of these candidate symbols, we can calculate the peak power of the signal in this particular symbol interval (referred to as a partial signal [8]).

In general, if the convolutional code has a $\nu$-bit memory, it has $2^{v}$ trellis states. The number of the resulting trellis states combined with $m_{\mathrm{ex}}$ external memories is thus given by $2^{\nu+m_{\mathrm{ex}}}$. Increasing $m_{\mathrm{ex}}$ enhances signal control capability and thus results in higher PAPR reduction. However, the price for this is the increased complexity at the transmitter as shown in [8].

2.2. Branch Metrics. Without loss of generality, it is assumed that an effective length of the pulse-shaping filter $g(t)$, denoted by $K_{s}$, is even. Here, we assume

$$
g(t) \approx 0, \quad \text { for } t<-\frac{K_{s}}{2} T_{s}, \quad \frac{K_{s}}{2} T_{s} \leq t .
$$




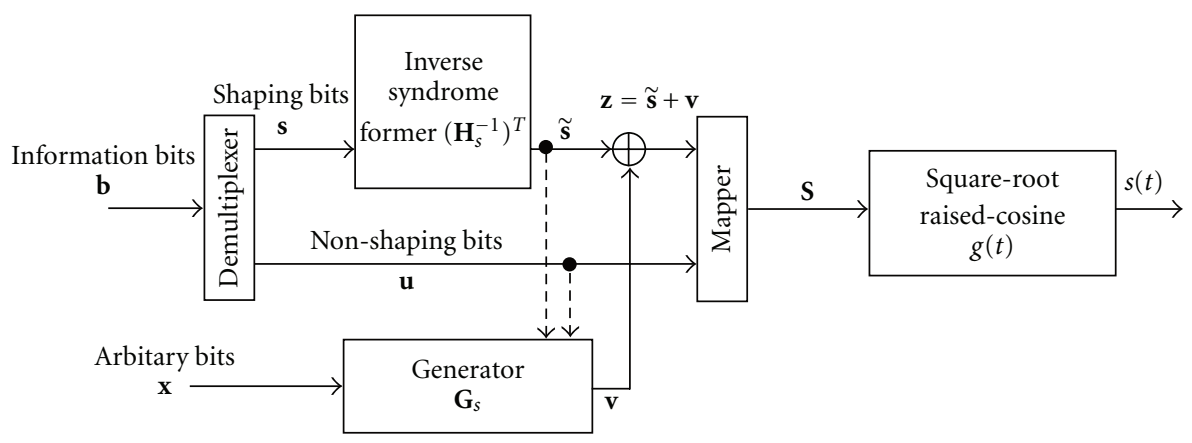

(a)

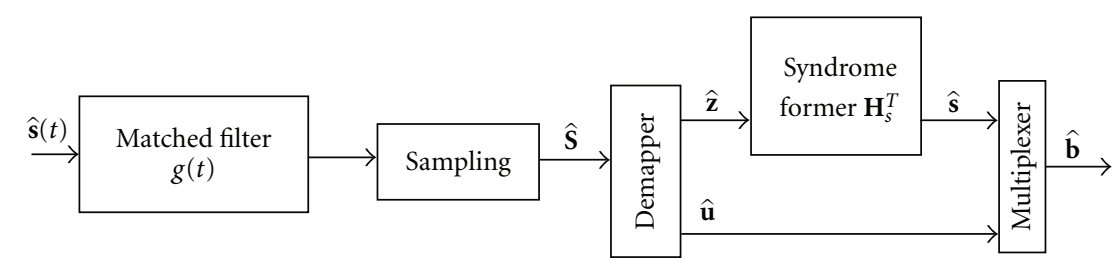

(b)

FIGURE 1: General trellis shaping system model. (a) Transmitter. (b) Receiver.

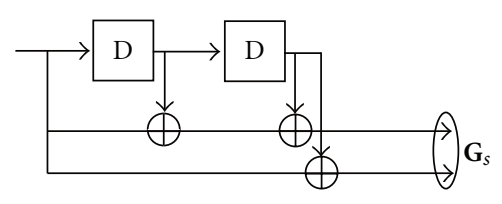

(a)

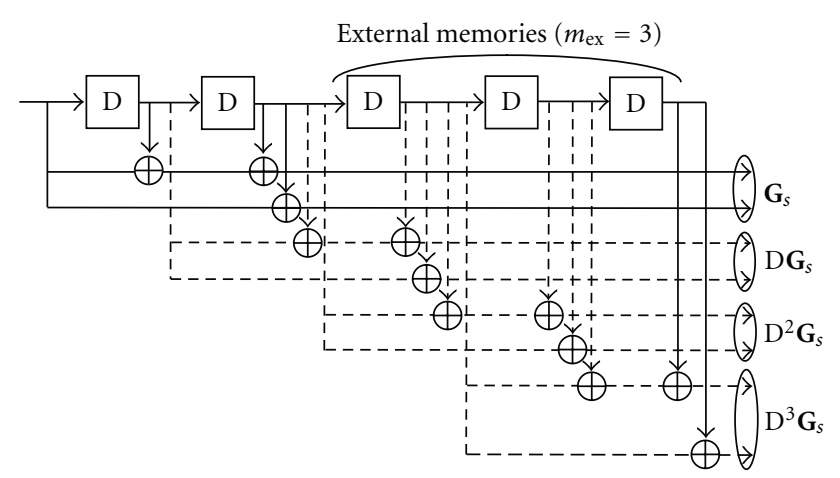

(b)

Figure 2: Construction example of code generator. (a) Code generator $(v=2)$. (b) Code generator with external memories $\left(m_{\mathrm{ex}}=3\right)$.

Due to the filtering process, the pulse-shaped signal for a given Nyquist symbol interval is determined by the $K_{s}$ consecutive symbols (i.e., $\left.\left\{S_{l-\left(K_{s}-1\right)}, \ldots, S_{l}\right\}\right)$.

The output signal sample $s[n]=s(n \Delta t)$ of the pulse shaping filter, where $\Delta t=T_{s} / N_{s}$, is expressed as

$$
s[n]=\sum_{k=-\infty}^{\infty} A(k) g[n-k],
$$

where $g[n] \triangleq g(n \Delta t)$,

$$
A(n)= \begin{cases}S_{n / N_{s}}, & n \text { is multiple of } N_{s}, \\ 0, & \text { otherwise }\end{cases}
$$

and $N_{s}$ is an oversampling factor.

In this paper, we use the limiter method [8] for branch metric calculation. In this method, we first set a predetermined threshold power $p_{\max }$. The branch metric is the total normalized instantaneous power that exceeds this threshold power. For a given normalized instantaneous power $p$, the branch metric $\mu(p)$ is expressed as

$$
\mu(p)=\max \left(p-p_{\max }, 0\right) .
$$

This ensures that the Viterbi decoder at the transmitter searches the codeword that minimizes the instantaneous power $|s[n]|^{2}$ that lies above a given threshold $p_{\max }$.

\section{Bit-Labeling Design for Trellis Shaping}

In the TS framework, the shaping and nonshaping bits are combined to form one PSK symbol, and bit labeling patterns determine how these bits are mapped onto a PSK symbol. Two commonly considered bit labelings in the communication system literature are Gray labeling and natural labeling; Gray labeling is formed such that any two neighboring signal points are different only in one bit. In the case of natural labeling, binary codes are allocated in a sequential order to the PSK signal points.

3.1. Desirable Bit Labeling from PAPR Reduction Perspective. In the TS system described in the previous section, the PAPR reduction capability strongly depends on the labeling of the 


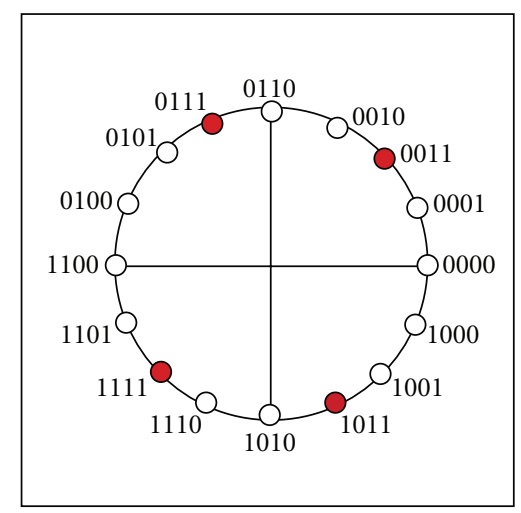

(a)

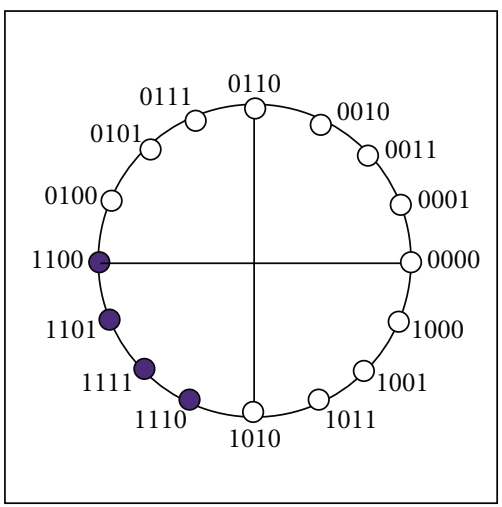

(b)

FIgURE 3: Two signal arrangement examples with Gray labeling 16-PSK where the nonshaping bits are 11. (a) The shaping bits choose the most significant bits (MSB). (b) The shaping bits choose the least significant bits (LSB).

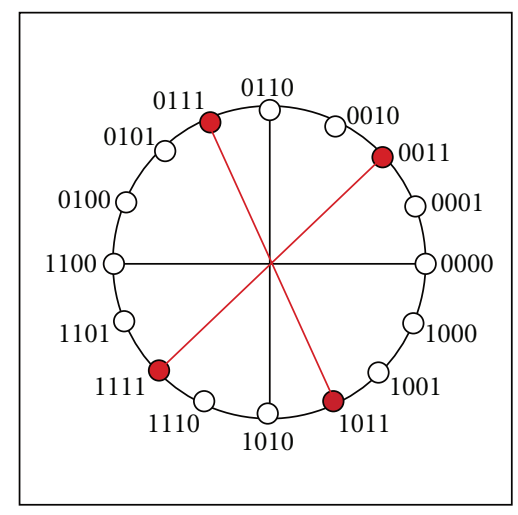

(a)

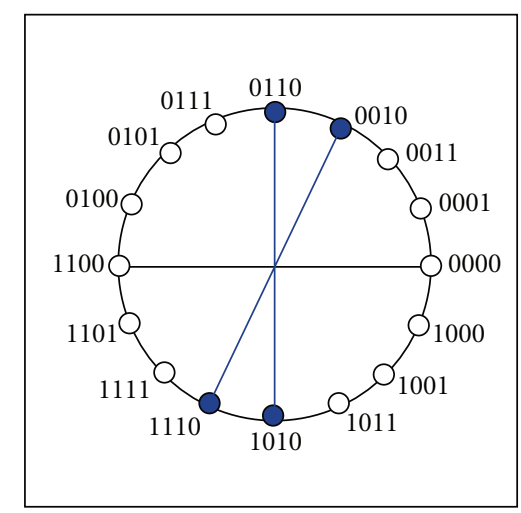

(b)

FIGURE 4: Two signal arrangement examples with Gray labeling 16-PSK where the nonshaping bits are fixed. In both cases, the shaping bits choose the most significant bits (MSB). (a) The nonshaping bits are 11. (b) The nonshaping bits are 10.

shaping bits. We first elucidate this fact by the following specific example.

Let us consider the case of 16-PSK, where each symbol carries $\log _{2} 16=4$ bits. Recalling that shaping bits are generated by (2), where the syndrome former is $\left(n_{s}-1\right) \times n_{s}$, only $n_{s}$ out of these 4 constituent bits will be controllable by the TS, suggesting that the PAPR control performance depends on the location where these $n_{s}$ bits are mapped.

Figures 3(a) and 3(b) show two examples of possible 16-PSK bit labelings, where $n_{s}=2$ and the two remaining nonshaping bits are given as 11 . Both of these two examples are based on Gray labeling, but the shaping bits in the left side choose the most significant bits (MSB) whereas those in the right side choose the least significant bits (LSB).

We can observe from these two examples that there is a wider range of selectable phases in the left bit labeling than in the right one: while the left bit labeling generates $2^{n_{s}}=4$ possible signal points almost equally spaced in the circle, all selectable points in the right bit labeling are located within a quadrant. In other words, there is more degree of freedom in selecting a phase of a symbol in the left labeling, and thus it can eliminate undesired consecutive phase patterns that will lead to the occurrence of high PAPR with higher probability.

Nevertheless, even if one adopts Gray labeling and two shaping bits select the MSB as in Figure 3(a), this does not guarantee that all the shaping bit patterns have this desirable (most separable) property. In Figures $4(\mathrm{a})$ and $4(\mathrm{~b})$, the different cases of nonshaping bit patterns are plotted with Gray labeling where nonshaping bits are given as 11 in the left side (same as Figure 3(a)) whereas they are given as 10 in the right side. As one can observe, in the case of the right side, the most separable property is not preserved; the two neighboring candidates are located in a similar phase so that the range of selectable phases is in effect reduced. For this reason, a bit labeling for efficient PAPR reduction is the one that has candidates equally-spaced over the constellation for any combination of nonshaping bits.

From this perspective, the most suitable bit labeling can be depicted in Figure 5 (where the shaping bits choose MSB). By inspection, the bit labeling in this figure is identified as a natural labeling, which may have the best PAPR reduction capability. 


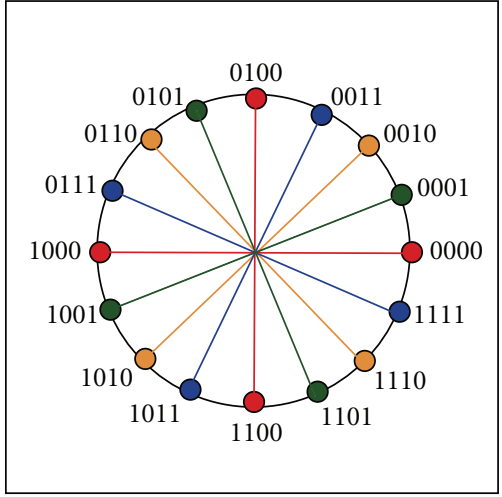

FIGURE 5: Bit labeling with equally spaced candidate signal points for any combinations of nonshaping bits. (The shaping bits choose MSB.)

3.2. Desirable Bit Labeling from BER Performance Perspective. In the uncoded system, it is well known that Gray labeling results in lower BER than other labelings in general since even if the transmitted symbol is received as the nearest neighbor signal point erroneously, it only causes one bit error. On the other hand, with natural labeling, one symbol error to the adjacent signal points may cause the maximum possible number of bit errors (e.g., $0000 \rightarrow 1111)$ in the worst case as observed in Figure 5.

As a figure of merit for BER performance, we consider the Hamming distance for a given bit labeling. Let $\mathbf{A}_{k}=$ $\left(A_{k, 0} A_{k, 1} \cdots A_{k, m-1}\right)$, where $A_{k, i} \in\{0,1\}$, denote a binary vector notation (labeling) of the $k$ th signal point of $M$-ary PSK symbol, where $m=\log _{2} M$. The Hamming distance between two distinct points $\mathbf{A}_{k}$ and $\mathbf{A}_{l}$ is then defined as

$$
d_{h}\left(\mathbf{A}_{k}, \mathbf{A}_{l}\right)=\sum_{i=0}^{m-1} d\left(A_{k, i}, A_{l, i}\right),
$$

where $d(x, y)=x \oplus y$ and $\oplus$ denotes an exclusive OR operation.

We define the average Hamming distance as the average number of bit errors caused by the symbol error from one signal point to its nearest neighbors

$$
d_{h, \mathrm{av}}=\frac{1}{M} \sum_{k=0}^{M-1} d_{h}\left(\mathbf{A}_{k}, \mathbf{A}_{k+1}\right),
$$

where $\mathbf{A}_{k}$ and $\mathbf{A}_{k+1}$ are a distinct pair of the two neighboring signal points. We also define, for each bit, the bitwise average Hamming distance as

$$
d_{b, i}=\frac{1}{M} \sum_{k=0}^{M-1} d\left(A_{k, i}, A_{k+1, i}\right),
$$

where the subscript $i$ refers to the bit position of the binary vector $\mathbf{A}_{k}$. This measure will be used for precise BER analysis of the TS later in Section 5. Apparently, we may express

$$
d_{h, \mathrm{av}}=\sum_{i=0}^{m-1} d_{b, i}
$$

If the symbol error from one signal point to its adjacent signal points occurs, the average number of bit errors associated with this symbol error event is determined by $d_{h, \mathrm{av}}$. For a high signal-noise ratio (SNR) region, this symbol error event is dominant. In this case, the probability of a symbol error for $M$-ary PSK $P_{M}$ is approximated as [14]

$$
P_{M} \approx 2 Q\left(\sqrt{\frac{2 E_{s}}{N_{0}}} \sin \frac{\pi}{M}\right),
$$

where $E_{s}$ is the signal energy per PSK symbol and $N_{0}$ is the one-sided power spectral density of an additive white Gaussian noise (AWGN) process. Since adjoining symbols differ in $d_{h \text {,av }}$ bits out of the $m$ constituent bits on average, the bit error rate can be expressed as

$$
P_{b} \approx \frac{d_{h, \mathrm{av}}}{m} P_{M}
$$

Therefore, smaller $d_{h, \mathrm{av}}$ results in better BER performance. By definition or by simple calculation, one may obtain

$$
d_{h, \mathrm{av}}= \begin{cases}1, & \text { for Gray labeling, } \\ 2-\frac{2}{M}, & \text { for natural labeling. }\end{cases}
$$

From (13), we observe that as $M$ increases the performance gap (asymptotic for high SNR) between Gray and natural labelings approaches a factor of 2 . Therefore, even though natural labeling is expected to have PAPR reduction capability superior to Gray labeling, it also has inferior uncoded BER performance compared to Gray labeling. This observation motivates us to propose a new labeling given in the next subsection.

3.3. A New Labeling: Double Gray Labeling. As we have seen, there is a trade-off between PAPR control capability (i.e., constellation separability within subset) and BER performance (average Hamming distance). The desirable labeling is the one that offers good constellation separability while maintaining small average Hamming distance.

In order to balance between PAPR control capability and BER degradation, we propose a new signal bit labeling which will be referred to as a double Gray labeling (DGray). The basic idea is to arrange the constellation such that both shaping bits and nonshaping bits form a Gray labeling property within their subsets, and to combine them such that the resulting constellation has small average Hamming distance. A 16-PSK example procedure for constructing this D-Gray labeling is illustrated in Figure 6(a). As shown in this figure, we first form a set of Gray labeling of size $n_{s}$ for shaping bits, and another Gray labeling set of size $m-n_{\mathrm{s}}$ for nonshaping bits. We then combine them such that any two neighboring signal points located in adjacent shaping bit subsets (A-B, B-C, C-D, and D-A) have at most two-bit differences as shown in Figure 6(b).

In this bit labeling, PAPR reduction capability is the same as that of natural labeling by comparing Figures 5 and 6(b). On the other hand, the Hamming distances between 


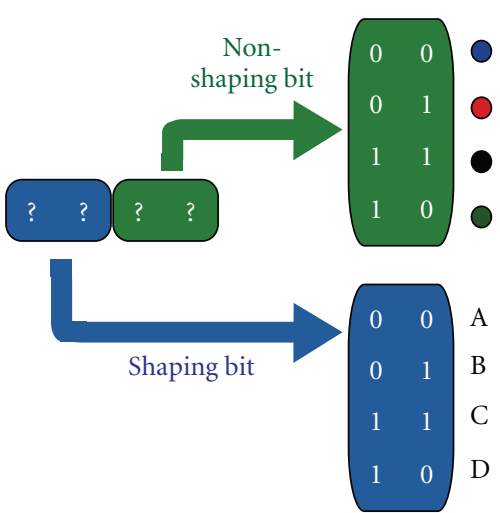

(a)

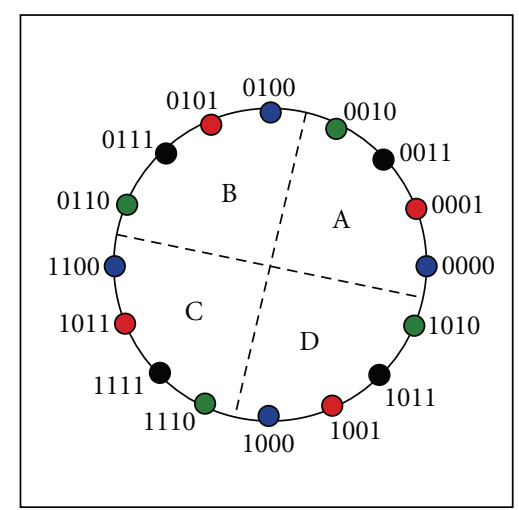

(b)

Figure 6: D-Gray labeling example for 16-PSK $\left(n_{s}=2\right)$. (a) Construction procedure. (b) D-Gray labeling where the shaping bits choose MSB (one of the regions A, B, C, and D).

neighboring signal points are guaranteed to be either 1 or 2 and thus

$$
\begin{aligned}
d_{h, \mathrm{av}} & =\frac{1 \cdot\left(M-2^{n_{s}}\right)+2 \cdot 2^{n_{s}}}{M} \\
& =1+\frac{2^{n_{s}}}{M}, \quad \text { for D-Gray labeling. }
\end{aligned}
$$

In the case of the above 16-PSK example, the average Hamming distance of this bit labeling is calculated to be 1.50 , which is smaller than that of natural labeling (i.e., 1.88). Furthermore, comparing (15) with (14) in the BER expression of (13), we observe that as $M$ increases for a fixed $n_{s}$, the BER of D-Gray labeling approaches that of Gray labeling, which is asymptotically half of that of natural labeling.

\section{Simulation Results}

In this section, we evaluate the performance of the TS with the proposed labeling as well as conventional labelings in terms of PAPR reduction capability and BER performance through computer simulations. We choose 32-PSK as an example in all the simulation results for trellis shaping, but similar results have been obtained for the PSK with other orders as long as $M \geq 16$. For fair comparison, we also evaluate the performance of unshaped 16-PSK system. This is because unshaped 16-PSK symbol and trellis-shaped 32PSK symbol carry the same amount of information bits (i.e., 4 bits), and thus they are equal in terms of bandwidth efficiency.

We use the complementary cumulative distribution function (CCDF) of the instantaneous power normalized by its average power for measuring PAPR reduction capability

$$
\operatorname{CCDF}\left(p_{0}\right)=\operatorname{Pr}\left[\frac{p}{p_{\mathrm{av}}}>p_{0}\right],
$$

where $p_{\mathrm{av}}$ denotes the average power and $p$ is the observed instantaneous power. The $\operatorname{CCDF}\left(p_{0}\right)$ thus indicates the probability that the normalized instantaneous power $p / p_{\mathrm{av}}$ exceeds a threshold power $p_{0}$.
We first determine the shaping parameters that will be used throughout the rest of this paper.

4.1. Simulation Setting. We choose a pulse shaping filter $g(t)$ as a commonly used square-root raised-cosine filter. We consider a strictly band-limited scenario, and thus we set the roll-off factor of $g(t)$ as $\alpha=0.1$. The effective duration of impulse response $K_{s}$ is chosen as 12 symbols. Subsequently, the number of external memories is chosen as $m_{\mathrm{ex}}=11$. The oversampling factor is chosen as $N_{s}=8$ for metric calculation in the TS process whereas we have oversampled more than 64 times upon CCDF evaluation of PAPR.

We consider the two cases where the numbers of shaping bits are $n_{s}=2$ and $n_{s}=3$. The memory length of the shaping encoder is chosen as $v=3$. The limiter method is adopted for metric calculation as described in Section 2.2. We note that the set of optimal shaping matrices, that is, $\mathbf{G}_{s}$, $\mathbf{H}_{s}^{T}$, and $\left(\mathbf{H}_{s}^{-1}\right)^{T}$, depends on the threshold parameter $p_{\max }$ in (7). Through exhaustive computer search, we found that a parameter $p_{\max }=1.33$ results in lowest value for a CCDF around $10^{-3}$. For each of the three labelings considered, the resulting sets of the three matrices are listed in Tables 1 and 2 for $n_{s}=2$ and 3 , respectively, where $D$ represents a delay element.

We note that the optimal parameter $p_{\max }$ and the resulting matrices depend on the target CCDF value at which the threshold PAPR is minimized, and they should be found by computer simulations.

4.2. PAPR Performance Comparison. Figure 7 shows the CCDF performances of the TS with Gray labeling where the shaping bit location is chosen as either MSB or LSB. We also plotted the CCDF of the unshaped 16-PSK.

From this result, we have the following remarks.

(i) For Gray labeling, allocating shaping bits to LSB results in poorer PAPR reduction performance compared to MSB bit allocation. This is consistent with the observation made in Figure 3. 
TABLE 1: The optimum parameters for $n_{s}=2$ with 32-PSK.

\begin{tabular}{|c|c|c|c|}
\hline labeling & Gray & Natural & D-Gray \\
\hline $\mathbf{G}_{s}$ & {$\left[\begin{array}{ll}1+D^{3} & 1+D^{2}+D^{3}\end{array}\right]$} & {$\left[1+D^{2}+D^{3} D^{2}\right]$} & {$\left[1+D^{3} 1+D+D^{3}\right]$} \\
\hline $\mathbf{H}_{s}^{T}$ & {$\left[\begin{array}{l}1+D^{2}+D^{3} \\
1+D^{3}\end{array}\right]$} & {$\left[\begin{array}{l}D^{2} \\
1+D^{2}+D^{3}\end{array}\right]$} & {$\left[\begin{array}{l}1+D^{2}+D^{3} \\
1+D^{3}\end{array}\right]$} \\
\hline$\left(\mathbf{H}_{s}^{-1}\right)^{T}$ & {$\left[\begin{array}{ll}D & 1+D\end{array}\right]$} & {$\left[\begin{array}{ll}1+D & 1\end{array}\right]$} & {$\left[\begin{array}{ll}1 & 1\end{array}\right]$} \\
\hline
\end{tabular}

TABLE 2: The optimum parameters for $n_{s}=3$ with 32-PSK.

\begin{tabular}{|c|c|c|c|}
\hline labeling & Gray & Natural & D-Gray \\
\hline $\mathbf{G}_{s}$ & 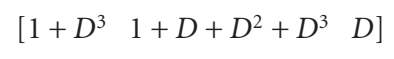 & {$\left[\begin{array}{lll}1+D+D^{2}+D^{3} & D+D^{2} & D\end{array}\right]$} & {$\left[1+D+D^{3} \quad 1+D^{2}+D^{3} D^{2}\right]$} \\
\hline $\mathbf{H}_{s}^{T}$ & {$\left[\begin{array}{ll}D & 0 \\
0 & D \\
1+D^{3} & 1+D+D^{2}+D^{3}\end{array}\right]$} & {$\left[\begin{array}{ll}D & 0 \\
0 & D \\
1+D+D^{2}+D^{3} & D+D^{2}\end{array}\right]$} & {$\left[\begin{array}{ll}D^{2} & 0 \\
0 & D^{2} \\
1+D+D^{3} & 1+D^{2}+D^{3}\end{array}\right]$} \\
\hline$\left(\mathbf{H}_{s}^{-1}\right)^{T}$ & $\left.\begin{array}{lll}1 & 0 & 0 \\
0 & 1 & 0\end{array}\right]$ & {$\left[\begin{array}{lll}1 & 0 & 0 \\
0 & 1 & 0\end{array}\right]$} & $\left.\begin{array}{lll}1 & 0 & 0 \\
0 & 1 & 0\end{array}\right]$ \\
\hline
\end{tabular}

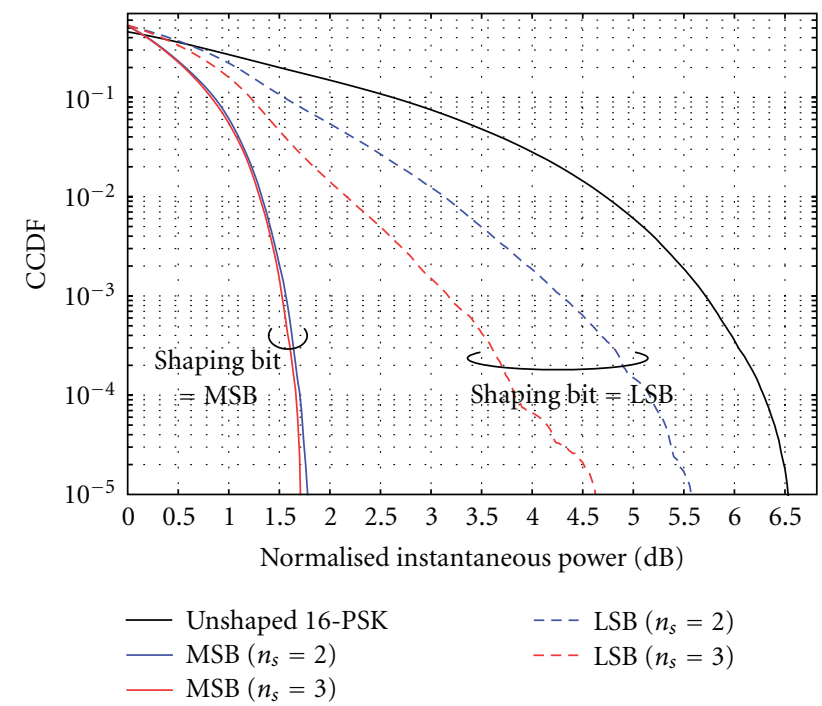

FIGURE 7: PAPR reduction performance for trellis-shaped 32-PSK with Gray labeling and different shaping bit allocation (MSB or LSB).

(ii) Performance of the TS with $n_{s}=3$ seems better than that with $n_{s}=2$. The difference is noticeable in the case where shaping bits are allocated to LSB.

(iii) In the case of MSB allocation, PAPR reduction from 16-PSK is more than $4.5 \mathrm{~dB}$ at $\mathrm{CCDF}=10^{-4}$.

Next, we compare the CCDF performances of the TS with Gray, natural and the proposed D-Gray labelings in Figure 8 (where the MSB set is chosen as shaping bits for

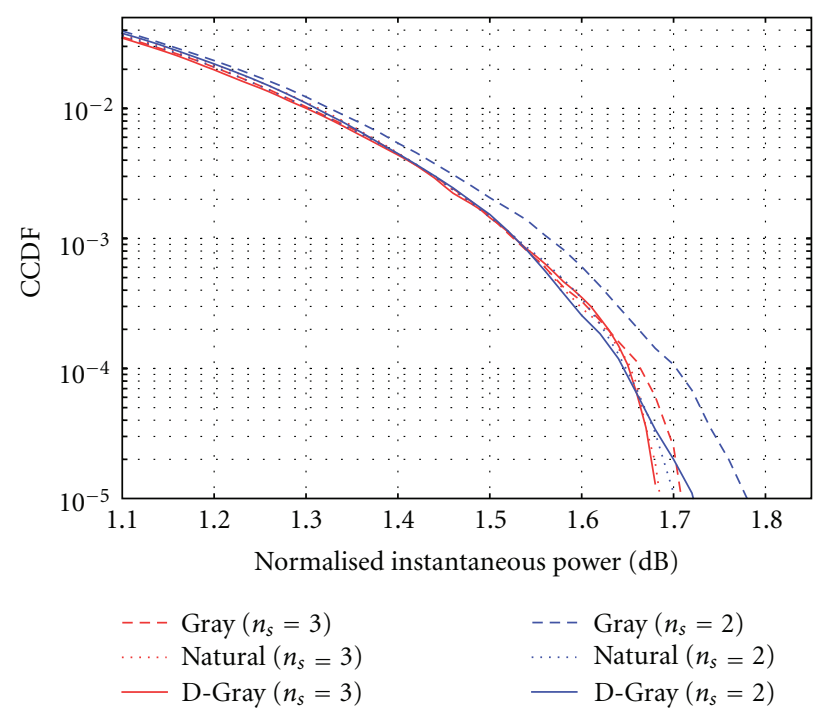

FIGURE 8: PAPR reduction performance with different labeling $\left(n_{s}=2,3\right)$.

all the labelings). It can be seen that the CCDF performance of the TS with natural labeling is better than that with Gray labeling. The D-Gray labeling can achieve the same performance as that of natural labeling as expected. No noticeable performance difference is observed for $n_{s}=2$ and $n_{s}=3$ except for the Gray labeling case.

4.3. BER Performance Comparison. The BER performances in an AWGN channel are shown in Figure 9. The horizontal axis is $E_{b} / N_{0}$ in $\mathrm{dB}$, where $E_{b}$ is the signal energy 


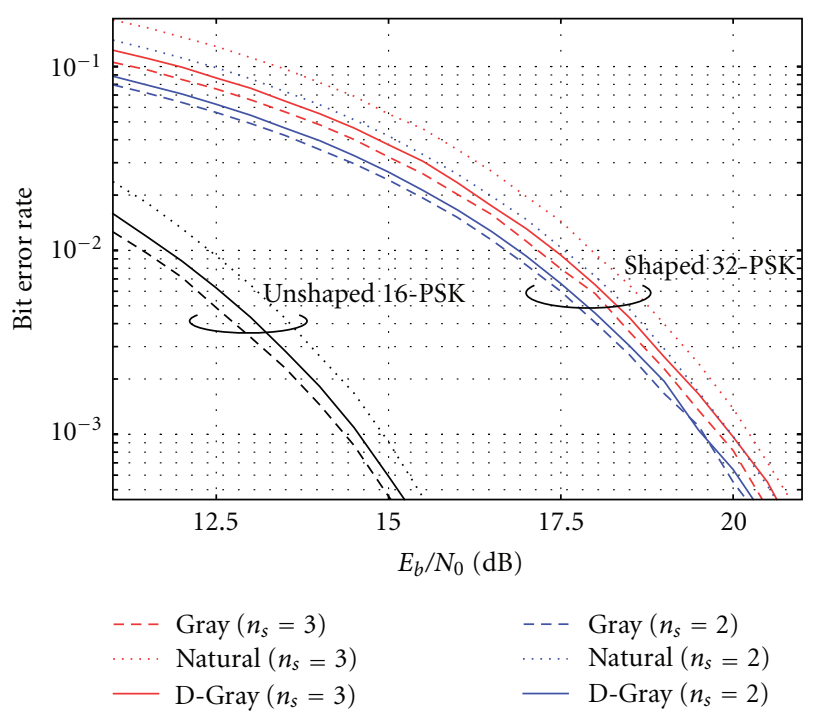

FIGURE 9: Bit error rate performance of the TS system with $n_{s}=2,3$ and different bit labelings.

per information bit. As expected, natural labeling performs worse than Gray labeling, but the BER of D-Gray labeling is close to that of Gray labeling. The gap of required SNR between the latter two is only within $0.1-0.2 \mathrm{~dB}$. It can be confirmed from (15) as well as the results in Figure 9 that the BER performance of D-Gray labeling with $n_{s}=2$ is always better than that with $n_{s}=3$ for high SNR region. The detailed analysis that explains the performance difference between $n_{s}=2$ and $n_{s}=3$ is given in Section 5 .

The price for the use of trellis shaping is its increased SNR required to achieve the same BER for a given $E_{b} / N_{0}$. When we compare the trellis shaped 32-PSK with unshaped 16-PSK, the BER performances of shaped systems are worse than that without shaping as observed in Figure 9. This performance gap is verified by the fact that the distance between neighboring signal points in 32-PSK is smaller than that in 16-PSK. The asymptotic loss in terms of the required $E_{b} / N_{0}$ for high SNR can be calculated from $P_{M}$ of (12) as

$$
\left(\frac{\sin (\pi / M)}{\sin (\pi / 2 M)}\right)^{2}=2\left(1+\cos \left(\frac{\pi}{M}\right)\right)
$$

which approaches $4(6 \mathrm{~dB})$ as $M$ increases. As we have seen previously, the use of the TS can reduce PAPR of about $4.5 \mathrm{~dB}$, which is lower than the above asymptotic loss. Nevertheless, it should be noted that the relative loss is considerably reduced when channel coding is employed. Furthermore, the benefit of PAPR reduction is more than just the SNR gain due to the increase of the transmit power. Reducing PAPR directly enhances the power amplifier efficiency; it significantly reduces power consumption of analog circuit at the transmitter with less heat dissipation. For long distance communications from battery-driven terminals, improvement of power amplifier efficiency may more than offset the associated penalty of increased $E_{b} / N_{0}$ requirement and signal processing complexity at the transmitter.

\section{Analysis of BER Performance for Uncoded TS}

Previous sections have demonstrated that the BER of the TS depends on the bit labelings and it has been shown that the average Hamming distance of a given bit labeling serves as a good estimate of the BER. Strictly speaking, however, the TS with uncoded system suffers from additional bit errors associated with the introduction of syndrome operation at the receiver [6]. Therefore, in this section, we theoretically analyze this performance degradation and make comparisons with the corresponding simulation results obtained in the previous section.

5.1. Approximate BER Analysis. Let us consider the case of Gray labeling with $n_{s}=3$ found in the previous section as a specific example. The received shaping bits $\mathbf{s}$ obtained after multiplication of $\mathbf{z}$ by $\mathbf{H}_{s}^{T}$ are expressed as

$$
\mathbf{s}=\mathbf{z H}_{s}^{T}
$$

or, from Table 2, we have

$$
\left[\begin{array}{ll}
s_{0} & s_{1}
\end{array}\right]=\left[\begin{array}{lll}
z_{0} & z_{1} & z_{2}
\end{array}\right]\left[\begin{array}{cc}
D & 0 \\
0 & D \\
1+D^{3} & 1+D+D^{2}+D^{3}
\end{array}\right],
$$

where $z_{i}$ and $s_{i}$ denote those with the $i$ th bit positions of the input and output of the symbol operation at the receiver. Now, let $z_{i, n}$ and $s_{i, n}$ denote those corresponding to the $n$th received PSK symbol. Then, we may express (19) as

$$
\begin{aligned}
& s_{0, n}=z_{0, n-1}+z_{2, n}+z_{2, n-3}, \\
& s_{1, n}=z_{1, n-1}+z_{2, n}+z_{2, n-1}+z_{2, n-2}+z_{2, n-3} .
\end{aligned}
$$

To obtain the correct value of $s_{0, n}$ at the receiver, we have to receive $z_{0, n-1}, z_{2, n}$, and $z_{2, n-3}$ correctly. Likewise, we have to receive $z_{1, n-1}, z_{2, n}, z_{2, n-1}, z_{2, n-2}$, and $z_{2, n-3}$ correctly for $s_{1, n}$. Hence, the error probability of $s_{0, n}$ is three times and that of $s_{1, n}$ is five times as large as those without shaping. Note that, strictly speaking, if the number of error bits is even, we can receive $\mathbf{s}$ correctly. However, even numbers of bit errors occur only with a negligible probability compared to a single bit error in a high $E_{b} / N_{0}$ region.

Let $P_{b, u_{i}}, P_{b, s_{i}}$, and $P_{b, z_{i}}$ denote the probabilities of bit error of nonshaping bit $u_{i}$, shaping bit $s_{i}$, and syndrome input $z_{i}$, respectively. Then $P_{b, s_{0}}$ and $P_{b, s_{1}}$ with respect to $n_{s}=3$ in the above example are expressed, respectively, as

$$
\begin{aligned}
& P_{b, s_{0}} \approx P_{b, z_{0}}+2 P_{b, z_{2}}, \\
& P_{b, s_{1}} \approx P_{b, z_{1}}+4 P_{b, z_{2}} .
\end{aligned}
$$

The average BER $P_{b \text {,av }}$ of this shaped 32-PSK for $n_{s}=3$ is calculated as

$$
\begin{aligned}
P_{b, \mathrm{av}} & =\frac{\left(P_{b, s_{0}}+P_{b, s_{1}}+P_{b, u_{0}}+P_{b, u_{1}}\right)}{4} \\
& \approx \frac{\left(P_{b, z_{0}}+P_{b, z_{1}}+6 P_{b, z_{2}}+P_{b, u_{0}}+P_{b, u_{1}}\right)}{4} .
\end{aligned}
$$


On the other hand, the average BER of unshaped 32-PSK $P_{b}$ can be expressed as

$$
P_{b}=\frac{\left(P_{b, z_{0}}+P_{b, z_{1}}+P_{b, z_{2}}+P_{b, u_{0}}+P_{b, u_{1}}\right)}{5} .
$$

From the above equations, we clearly observe that $P_{b \text {,av }}>P_{b}$, indicating that the average BER of the above trellis shaping is worse than that of unshaped 32-PSK system.

Let $A_{i}$ denote the $i$ th bit of the binary vector representation of the PSK symbol A. We can then express the error rate of this bit as

$$
P_{b, A_{i}} \approx d_{b, i} P_{M}
$$

where $d_{b, i}$ is the bitwise average Hamming distance of the $i$ th bit position defined in (10). In our TS design, the shaping bits are arranged as MSB. Consequently, $z_{i}$ and $u_{i}$ are related to $A_{i}$ as

$$
\left(\begin{array}{lllll}
z_{0} & z_{1} & z_{2} & u_{0} & u_{1}
\end{array}\right)=\left(\begin{array}{lllll}
A_{0} & A_{1} & A_{2} & A_{3} & A_{4}
\end{array}\right) .
$$

We can now calculate $P_{b \text {,av }}$ of shaped 32-PSK $\left(n_{s}=3\right)$ with Gray labeling as

$$
\begin{aligned}
P_{b, \mathrm{av}} & \approx \frac{\left(P_{b, z_{0}}+P_{b, z_{1}}+6 P_{b, z_{2}}+P_{b, u_{0}}+P_{b, u_{1}}\right)}{4} \\
& \approx \frac{\left(d_{b, 0}+d_{b, 1}+6 d_{b, 2}+d_{b, 3}+d_{b, 4}\right) P_{M}}{4} \\
& =\frac{(2 / 32+2 / 32+6(4 / 32)+8 / 32+16 / 32) P_{M}}{4} \\
& =\frac{52}{128} P_{M} .
\end{aligned}
$$

Likewise, in the case of Gray labeling with $n_{s}=2$, referring to Table 1, the shaping bits $\mathbf{s}$ are expressed as

$$
s_{0, n}=z_{0, n}+z_{0, n-2}+z_{0, n-3}+z_{1, n}+z_{1, n-3} .
$$

Therefore, we obtain

$$
P_{b, s_{0}} \approx 3 P_{b, z_{0}}+2 P_{b, z_{1}}
$$

and the average BER $P_{b \text {,av }}$ of shaped 32-PSK in this case is calculated as

$$
\begin{aligned}
P_{b, \mathrm{av}} & =\frac{\left(P_{b, s_{0}}+P_{b, u_{0}}+P_{b, u_{1}}+P_{b, u_{2}}\right)}{4} \\
& =\frac{\left(3 P_{b, z_{0}}+2 P_{b, z_{1}}+P_{b, u_{0}}+P_{b, u_{1}}+P_{b, u_{2}}\right)}{4} .
\end{aligned}
$$

We again observe that $P_{b, \mathrm{av}}>P_{b}$ in this case. The corresponding expression of $P_{b \text {,av }}$ with respect to $P_{M}$ can be obtained following (26) in a straightforward manner.

Table 3 summarizes the probability $P_{b \text {,av }}$ for each labeling with both $n_{s}=2$ and $n_{s}=3$ as well as $P_{b}$ of unshaped system. We note that for all the labelings compared, $P_{b \text {,av }}$ with $n_{s}=3$ is larger than that with $n_{s}=2$, which suggests that the burst error at the syndrome operation is more significant for $n_{s}=3$ than that for $n_{s}=2$.

Finally, substituting approximate symbol error probability of (12) into $P_{M}$ in the above expression, the approximate BER performance can be calculated.
TABLE 3: Average BER expressions of unshaped and trellis shaped 32-PSK systems with respect to the symbol error rate $P_{M}$. (For unshaped system with D-Gray labeling, $n_{s}=3$ is used for calculation.)

\begin{tabular}{llll}
\hline Labeling & Gray & Natural & D-Gray \\
\hline$n_{s}=2$ & $\frac{38}{128} P_{M}$ & $\frac{70}{128} P_{M}$ & $\frac{46}{128} P_{M}$ \\
$n_{s}=3$ & $\frac{52}{128} P_{M}$ & $\frac{102}{128} P_{M}$ & $\frac{60}{128} P_{M}$ \\
unshaped & $\frac{1}{5} P_{M}$ & $\frac{31}{80} P_{M}$ & $\frac{1}{4} P_{M}$
\end{tabular}

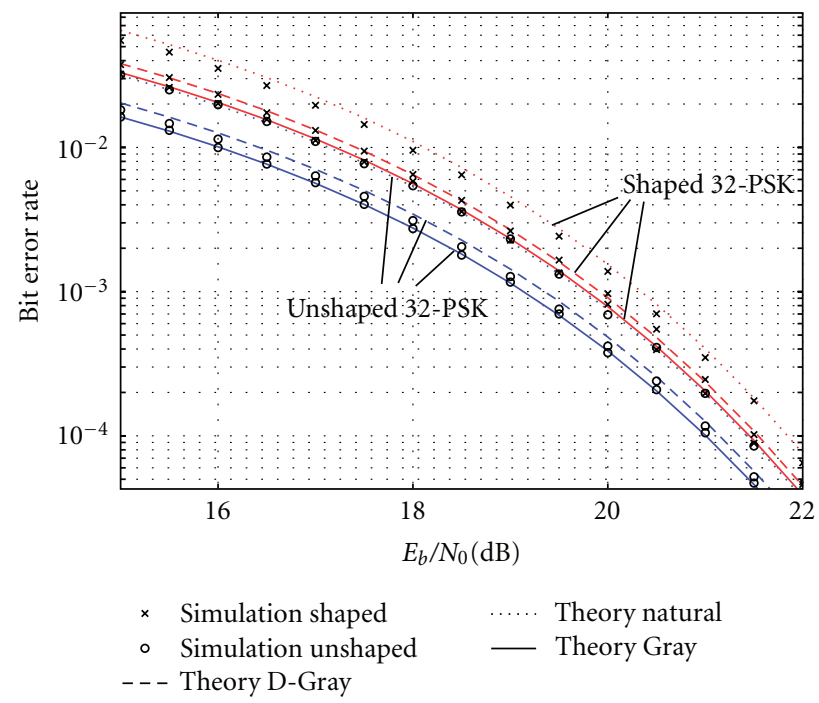

FIGURE 10: Bit error rate performances of the 32-PSK TS with $n_{s}=3$ and the three bit labelings. The corresponding unshaped 32-PSK performances are also plotted for comparison.

5.2. Numerical Comparison. Figure 10 compares the theoretical BER expressions and those obtained by simulations for trellis-shaped 32-PSK system with $n_{s}=3$ and the three bit labelings considered in this paper. The results for unshaped 32PSK with the three labelings are also plotted for comparison. Both simulation and analytical results show good agreement for high SNR region.

The major gap between shaped and unshaped systems in terms of the required $E_{b} / N_{0}$ is that $E_{s}=m E_{b}$ for unshaped $M$-PSK whereas $E_{s}=(m-1) E_{b}$ for shaped $M$-PSK considered in this paper due to the reduction of information rate associated with TS. Therefore, for a given $E_{b} / N_{0}$, there is a loss in terms of $E_{s} / N_{0}$ as $10 \log _{10}(m /(m-1))$. In the case of 32-PSK where $m=5$, this loss turns out to be $0.97 \mathrm{~dB}$. This loss, however, becomes negligible as $M$ increases.

\section{Conclusions and Future Work}

The bit labeling of signal constellation has nontrivial influence on the PAPR reduction capability of the TS scheme. 
In this paper, we have investigated several bit labelings and illuminated that the PAPR reduction capability is related largely to the range of selectable phases. The commonly used bit labeling, that is, a Gray labeling, is found not to be suitable in this perspective while the natural labeling offers a better PAPR reduction. However, since the use of the latter labeling poses some SNR loss (in an uncoded case), we have proposed an alternative new labeling called D-Gray labeling that exhibits the same PAPR characteristic as that of the natural labeling but can achieve the BER performance close to that of a Gray labeling. The BER performance of the TS system with the considered bit labelings has been also analyzed theoretically. Even though we have considered our specific TS system, we note that the analysis is applicable to general uncoded TS systems in a straightforward manner.

Throughout this paper, we have only considered the performance of the system over an AWGN channel. Considering the fact that single-carrier systems are being considered in the high-speed mobile communication system applications, their performances over frequency-selective fading channels should be also investigated. In this case, the use of channel equalization is necessary. The considered trellis shaping is also applicable to block transmission with cyclic prefix together with FDE as in [1], but this extension is nontrivial since the symbol extension by cyclic prefix typically introduces discontinuity and thus results in increase of the peak power similar to CPM case [5]. This issue, together with the performance evaluation of the TS system over frequencyselective fading channels is beyond the scope of this paper and left as our future work.

Finally, in practice, the use of channel coding is necessary to guarantee reliable communications, and we note that introduction of channel coding may complicate a design criterion for bit labeling. This issue should also be investigated.

\section{Acknowledgments}

This paper was supported in part by the Strategic Information and Communications R\&D Promotion Programme (SCOPE), the Ministry of Internal Affairs and Communications, Japan.

\section{References}

[1] D. Falconer, S. L. Ariyavisitakul, A. Benyamin-Seeyar, and B. Eidson, "Frequency domain equalization for single-carrier broadband wireless systems," IEEE Communications Magazine, vol. 40 , no. 4 , pp. 58-66, 2002.

[2] S. L. Miller and R. J. O'Dea, "Peak power and bandwidth efficient linear modulation," IEEE Transactions on Communications, vol. 46, no. 12, pp. 1639-1648, 1998.

[3] H. G. Myung, J. Lim, and D. J. Goodman, "Peak-to-average power ratio of single carrier FDMA signals with pulse shaping," in Proceedings of the 17th International Symposium on Personal, Indoor and Mobile Radio Communications (PIMRC '06), September 2006.

[4] C. Ciochina, D. Castelain, D. Mottier, and H. Sari, "New PAPR-preserving mapping methods for single-carrier FDMA with space-frequency block codes," IEEE Transactions on Wireless Communications, vol. 8, no. 10, pp. 5176-5186, 2009.
[5] T. Buzid and M. Huemer, "Single carrier transmission with frequency domain equalization (SC/FDE) system with a PAPR of unity," in Proceedings of the 11th International Conference on Advanced Communication Technology (ICACT '09), pp. 459462, February 2009.

[6] G. D. Forney Jr., "Trellis shaping," IEEE Transactions on Information Theory, vol. 38, no. 2, pp. 281-300, 1992.

[7] I. S. Morrison, "Trellis shaping applied to reducing the envelopefluctuations of MQAM and band-limited MPSK," in Proceedings of the International Conference on Digital Satellite Communications (ICDSC'92), pp. 143-149, May 1992.

[8] M. Tanahashi and H. Ochiai, "Near constant envelope trellis shaping for PSK signaling," IEEE Transactions on Communications, vol. 57, no. 2, pp. 450-458, 2009.

[9] E. Agrell, J. Lassing, E. G. Ström, and T. Ottosson, "On the optimality of the binary reflected gray code," IEEE Transactions on Information Theory, vol. 50, no. 12, pp. 3170-3182, 2004.

[10] E. Agrell, J. Lassing, E. G. Ström, and T. Ottosson, "Gray coding for multilevel constellations in Gaussian noise," IEEE Transactions on Information Theory, vol. 53, no. 1, pp. 224235, 2007.

[11] N. S. Muhammad and J. Speidel, "Joint optimization of signal constellation and bit labeling for bit-interleaved coded modulation with iterative decoding," IEEE Communications Letters, vol. 9, no. 9, pp. 775-777, 2005.

[12] X. Li and J. A. Ritcey, "Bit-interleaved coded modulation with iterative decoding," IEEE Communications Letters, vol. 1, no. 6, pp. 169-171, 1997.

[13] D. Torrieri and M. Valenti, "Constellation labeling maps for low error floors," IEEE Transactions on Wireless Communications, vol. 7, no. 12, pp. 5401-5407, 2008.

[14] J. G. Proakis, Digital Communications, McGraw-Hill, New York, NY, USA, 4th edition, 2000. 